\title{
An unexpected finding in a patient with a DDDR-PM
}

\author{
A. Bohm • R. G. Kiss • G. Z. Duray
}

Published online: 4 April 2014

(C) The Author(s) 2014. This article is published with open access at Springerlink.com

We noticed an unprecedented sensing error: after a correctly sensed ventricular extrasystole followed by a correctly timed atrial stimulus, a ventricular impulse occurs that is not sensed, then the delivery of a ventricular stimulus can be observed at the set AV delay (225 ms) (Fig. 1). The subsequent stimuli appear to be sensed correctly, judging from the lack of any atrial or ventricular spikes.

Fig. 1 Ventricular sensing failure, when the QRS is narrow

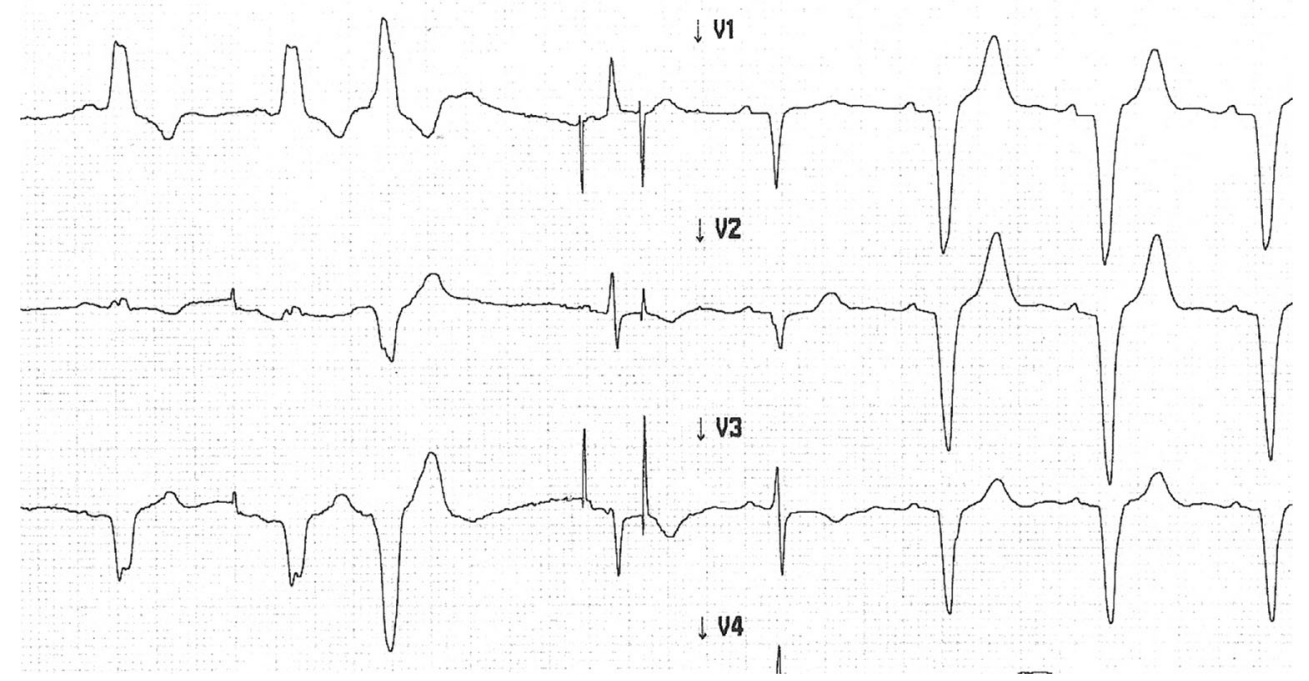

A. Bohm $(\bowtie) \cdot$ R. G. Kiss $\cdot$ G. Z. Duray

Department of Cardiology, Military Hospital, Róbert K körút 44,

Budapest 1134, Hungary

e-mail: abohm@freemail.hu 


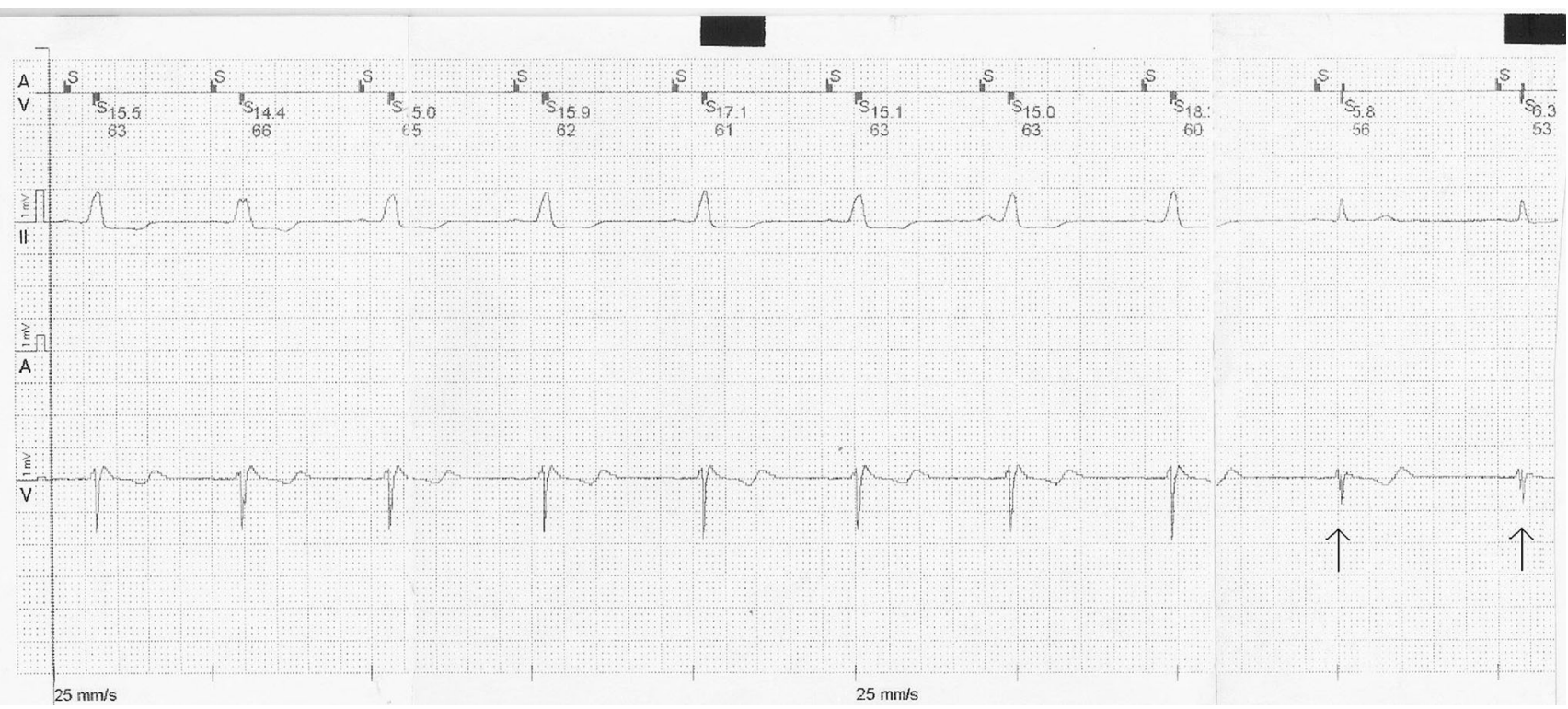

Fig. 2 Bundle branch dependent $\mathrm{R}$ wave amplitude

IEGM scans eventually revealed the cause of the unexpected sensing error: while the R-wave amplitude exceeded $15 \mathrm{mV}$ with a bundle branch block, we sensed a significantly lower R-wave amplitude with a narrow QRS (5.8-6.3 $\mathrm{mV}$ in the last two QRS) (Fig. 2). At a sensitivity of $5 \mathrm{mV}$, the pacemaker only intermittently sensed a narrow QRS. We have now lowered the ventricular sensitivity to an appropriate level.

Open Access This article is distributed under the terms of the Creative Commons Attribution License which permits any use, distribution, and reproduction in any medium, provided the original author(s) and the source are credited. 